\title{
LAS POLÍTICAS \\ EDUCATIVAS EN LA \\ REFLEXIÓN FILOSÓFICA
}

Darwin Reyes

Universidad Politécnica Salesiana - Quito

\section{Introducción}

Política, filosofía y educación convergen en el punto del análisis de la construcción de lo humano y de la producción del saber que la humanidad ha elaborado en sus procesos sociales y culturales. Temas como la libertad, la identidad y la alteridad, la ética, la sociedad, lo que se debe transmitir a las futuras generaciones, la problemática de cómo conoce el ser humano, la pregunta por el cómo transmitir conocimientos para que el saber humano perdure, son problemas que tanto la filosofía, como la política y los estudios sobre la educación trabajan desde diferentes perspectivas.

Dichas temáticas ponen al proceso educativo de una sociedad frente a la necesidad de definir políticas para educar a las personas que pertenecen a dicha comunidad. ¿Puede la filosofía aportar en la construcción de dichas políticas? ¿Es posible pensar desde la construcción filosófica las políticas educativas?, o ¿es mejor abandonar tal empresa por ser un imposible?

No hay proceso pedagógico que se ejecute en una realidad específica sin políticas educativas sustentadas en 
construcciones conceptuales, muchas de ellas estrictamente filosóficas. La conceptualización sobre lo que entendemos por lo "humano" o por la "sociedad que queremos" constituyen pre-juicios sobre los cuales se levantan políticas educativas que determinan metas específicas que se quieren lograr en la educación; prioridades que se establecen; contenidos seleccionados consciente o inconscientemente, y mediaciones que se van a utilizar en el proceso de aprendizaje.

\section{La educación como construcción de lo humano}

Uno de los aportes significativos de la filosofía a las políticas educativas se concentra en el debate que la filosofía ha mantenido sobre el problema de lo humano. De las conclusiones que se saquen de dicho debate dependerán en gran medida las aplicaciones políticas que se requieran para la educación.

La relación entre lo humano y el acto de aprender es ontológico: El humano se hace en la medida que vive experiencias y prácticas educativas; la aventura histórica de lo humano ha sido apoyada desde los procesos de aprendizaje. El ser humano es un ser que no está acabado, porque es un ser temporal. Es un ser que se está haciendo y no dejará de hacerse hasta su muerte. Es en este sentido ontológico que el proceso de aprendizaje es fundamental para la humanización, para lograr el advenimiento de lo humano en cada sujeto que se constituye dentro de la especie.

El ser humano es un proyecto inacabado, porque está en el tiempo, y ese tiempo lo determina en lo que es; en otras palabras, no puede ser sin temporalidad. Ahora bien, si el ser es temporal, al transcurrir las experiencias en el tiempo, se va construyendo la historicidad. La temporalidad es el fundamento de la historicidad del ser. 
Así como el ser humano, el modo de comprender humano también es histórico; este modo de comprender lo hemos denominado tradicionalmente "experiencia" o "aprendizaje". Todo aprendizaje es expresión de la vida. Vida y saber forman una unidad de interrelación mutua.

La humanización como historicidad de las personas sólo es posible desde el apropiamiento del conocer lo que otras generaciones han dejado y del entender la posibilidad de la innovación que cada sujeto histórico puede hacer. No existe una persona que pueda vivir sin relación con los otros, sin comunidad, sin sociedad. La persona se realiza dentro de un grupo o comunidad en relación permanente con las otras personas. Por ello es importante superar el egoísmo e individualismo potenciado en el sistema capitalista y avanzar hacia una forma de entender a los seres humanos desde la solidaridad social.

No solamente se trata de abrirnos a otras personas que conocemos, sino que somos sujetos sociales que juntos construimos el mundo que queremos. Somos responsables de lo que acontece en nuestra comunidad.

Entender al ser humano en inter-relación con los demás implica rechazar la autosuficiencia del yo individual y construir el encuentro con la naturaleza y con los demás para lograr una sociedad solidaria. No podemos pensar en una educación que no trabaje las dimensiones del "otro". La dimensión más profunda de los seres humanos es su relación con los otros: el ser humano es en cuanto es con los demás y se construye en relación "a" los demás. En definitiva, los procesos de educación son procesos de relación. Esas relaciones entre humanos que nos muestra semejantes en nuestra humanidad. Desde allí podemos partir para construirnos como una sociedad que no excluye, sino que incluye.

La dimensión del otro debe ser asumida en los sistemas de educación nacional. Es apremiante superar al 
individuo promocionado por tendencias utilitaristas que construyen modelos de exclusión en base a las diferencias. Es necesario construir modelos de inclusión en base a las diferencias. Los seres humanos compartimos la identidad de humanos, pero no somos iguales. Somos diferentes y desde esas diferencias queremos construir sociedades incluyentes.

\section{La educación como acto político de transformación social}

La educación es una construcción social, no va divorciada de lo que la sociedad es y quiere ser a futuro, y está en íntima conexión con la relación que establecemos con los demás, con los diferentes grupos sociales y culturales de nuestra colectividad. ${ }^{1}$

La propuesta educativa siempre implica una permanente acción que se define en el ejercicio de la construcción social y no fuera de ella. No se hace educación sin un proyecto de sociedad, sin un proyecto político que piense el futuro. Las fuerzas sociales han de colocar el eje del debate sobre la educación no solamente en los contenidos, sino en la concepción de un proyecto político, de una sociedad que busca su propio camino.

$\mathrm{Y}$ es en este punto, las sociedades que buscan su propio camino, que surge una contradicción, un límite en el pensar la educación: adultos de sociedades excluyentes, injustas socialmente, de pésima distribución de la rique$\mathrm{za}$, de atentados permanentes contra el medio ambiente, de construcciones sociales racistas, clasistas, nos proponemos "enseñar" desde concepciones pedagógicas y educativas nuevas; cómo dichos adultos y adultas que hemos vivido en sociedades de discriminación, divisiones entre los que tienen y los que no tienen; extrema riqueza y extrema pobreza, en la dicotomías de superior - inferior, amo - 
peón, queremos enseñar a nuestros niños, niñas, jóvenes y a nosotros mismos la posibilidad de ver al otro como un semejante, la posibilidad de una sociedad incluyente, justa y solidaria, la posibilidad de otro tipo de interrelaciones y sensibilidades.

¿Es posible eso? ¿Realmente creemos que podemos hacerlo? Consideramos que lo primero es reconocer los serios límites de tal empresa. Y los límites somos nosotros como sociedad. Somos parte de los límites y somos parte de la propuesta de no ser límites. Si no tenemos en cuenta esto, podemos estar clasificando a la sociedad y a la educación en buenos y malos. Por supuesto que la mismidad nos va a llevar a pensar que estamos del lado de los buenos. El problema se plantea como una paradoja insalvable. Yo que soy un límite, me planteo romper esos límites, y eso nos lleva a romper nuestro ser mismo. El sujeto no puede construir su propio ser sin romper las construcciones de lo que entiende por ser.

Pensemos en un momento cómo nos hemos construido en la educación que hemos recibido. Dominó, y sospechamos que aún lo hace, la vieja tradición de concebir a los procesos de la educación formal desde estructuras conceptuales conductistas, con el parámetro básico: estímulo-respuesta, en donde el estímulo está dado desde la violencia y la respuesta se anhela que sea el aprendizaje. Este esquema se reproduce en los sistemas formales con una manifestación cotidiana, aceptada dentro del aula y rechazada en los discursos pedagógicos y foros públicos. Así tenemos que el grito, la agresión, el insulto, la amenaza, el chantaje y otras formas se quieren usar como estímulo y la respuesta anhelada es el aprendizaje de tal o cual contenido. Esto no es aceptado en ningún espacio teórico, ningún docente aceptaría que es posible este manejo conceptual para enseñar. Sin embargo, en el aula, las relaciones docente-alumno siguen manejadas desde estos 
parámetros de poder y dominación, de esquemas violentos y discriminantes. Nos parece clave construir reflexiones teórico-conceptuales que analicen las estructuras de estos sistemas de violencia y dolor. Mucho más importante en los espacios campesinos y periféricos urbanos de nuestros países latinoamericanos.

Además, es interesante analizar los recuerdos que las personas tienen sobre su propio proceso educativo formal. En altos porcentajes dichos recuerdos están atados a estructuras síquicas dolorosas. Eso hace que los sistemas de reproducción del dolor se conviertan en repeticiones de generación en generación marcando la formación de las personas. Es necesario tomar conciencia de esos procesos para poner un "alto" a las reproducciones del acto educativo como expresión de dolor: "la letra con sangre entra" en versiones sofisticadas, en nuevas tecnologías de imposición y dominación de las almas y de los cuerpos.

Podemos plantearnos un ejercicio. Recordemos nuestra historia educativa. Veamos qué está en juego cuando recorremos nuestra memoria de los procesos de aprendizaje. Iniciemos por el dolor de la inserción escolar. Un gran porcentaje de esa memoria que tenemos de nuestros procesos educativos está atada al dolor. Mucho más en sectores indígenas, sectores periféricos urbanos, sectores negros y campesinos. ¿Cómo construir una educación diferente, si en lo más profundo, en nuestra memoria histórica como individuos, en nuestro inconsciente, están grabados los sistemas de dolor? ¿No será que a la final nos vencen esas memorias estructuradas desde el sufrimiento y se imponen en nuestras prácticas educativas? ¿No será que el acto educativo se ha construido como un acto de reproducción de nuestros sistemas de sufrimiento y dolor? No sólo es un debate en el sentido de la educación como reproducción social y cultural, sino que reproducimos 
un sistema de sociedad sufriente. Pensemos en algunos "modelos" que establecen la educación y la sociedad.

En definitiva, queremos expresar que nosotros somos un límite serio en la construcción del acto educativo como actores de lo humano, pero a la vez somos la esperanza de que esas formas educativas alternativas se ejecuten. Esto me recuerda el mito del médico herido. Es el herido el que cura al otro. Es esa herida que está en mí la que genera esperanza de encontrar la sanación para el otro y para mí también. No podemos abandonar el proyecto de la educación en la tierra de los imposibles. El camino puede ser arduo, mas todo está iluminando en esa dirección.

\title{
3. La comunidad como constructora de los procesos educativos
}

\author{
"Nadie educa a nadie, todos nos educamos" \\ Paulo Freire
}

Pensar que sólo la escuela educa a los niños y niñas es un acto de "inocencia" e ignorancia. Al pensar en educación estamos trabajando no sólo la escuela y el colegio, sino el barrio, las organizaciones, la familia, las comunidades, la ciudad, los grupos políticos, la sociedad civil.

La educación tiene como escenario la sociedad en la que se inscribe el proyecto de aprendizaje. Por ello es necesario que las políticas educativas no solamente estén orientadas a la educación formal, sino a la educación dentro de un proyecto social.

Los actores del proceso educativo son todos aquellos que están en la sociedad, y el compromiso no es únicamente para la escuela y la burocracia estatal de la que depende la educación, sino que la aventura de la humanización es de varios actores. Analizaremos a conti- 
nuación algunos actores, sin querer decir que son todos los actores posibles, sino sólo una muestra de ellos.

\subsection{Políticas de Estado}

"Educación: un compromiso de todos para cambiar la historia" Ministerio de Educación y Cultura

El Estado, como institución que concretiza el acuerdo social de un pueblo, es un actor importante dentro de la elaboración de políticas para la educación y dentro del debate sobre las dimensiones filosóficas sobre las cuales queremos construir el proceso educativo.

Hasta el momento, el Estado ha sido un actor débil en la definición de políticas públicas. Se ha visto marcado por una fuerte privatización de la educación abandonando su estructura fiscal para la educación formal y entregándola, bajo leyes preferenciales, a los actores privados. La educación ha estado conceptualizada y prejuiciada por la diferenciación de educación pública y educación privada. Es más, las instancias que rigen y regulan los procesos educativos son diferentes y autónomas algunas de ellas, casi ministerios paralelos: está la CONFEDEC para instituciones educativas católicas; La DINEIB para instituciones educativas de los pueblos y nacionalidades indígenas, y las direcciones de educación de los municipios que tienen a su haber instituciones de educación formal como escuelas y colegios.

Es clave el debate que la sociedad construya sobre lo que el Estado debe manejar en sus políticas de educación para todos y bajo qué fundamentos filosóficos lo haga. En el 2006 se elaboró un plan decenal ${ }^{2}$ para tener una referencia desde la cual trabajar: parte de un análisis de la realidad educativa, luego se plantean objetivos del sistema educativo ecuatoriano, posteriormente se trabaja la visión, misión, finalidad y propósito del sistema educa- 
tivo, para finalmente presentar ocho políticas para trabajar. Cada política contiene justificación y proyectos definidos con sus respectivos componentes, actividades, tiempos, presupuestos. A continuación presentamos un resumen de las ocho políticas definidas como prioritarias.

P. 1: Universalización de la educación infantil de 0 a 5 años de edad.

P. 2: Universalización de la educación general Básica de primero a décimo años.

P. 3: Incremento de la matrícula en el Bachillerato hasta alcanzar al menos el 75\% de la población en la edad correspondiente.

P. 4: Erradicación del analfabetismo y fortalecimiento de la educación Alternativa.

P. 5: Mejoramiento de la infraestructura física y el equipamiento de las instituciones educativas.

P. 6: Mejoramiento de calidad y equidad de la educación e implementación del sistema nacional de evaluación.

P. 7: Revalorización de la profesión docente, desarrollo profesional, condiciones de trabajo y calidad de vida.

P. 8: Aumento del $0.5 \%$ anual en la participación del sector educativo en el PIB hasta alcanzar al menos el 6\%.

Analizada la propuesta, surge la inquietud de que todas las políticas están dirigidas a lo básico, a lo que se presupone debe estar resuelto. Un planteamiento de este tipo de políticas devela la sociedad que tenemos, la fuerte discriminación que existe y las dificultades que a lo largo del tiempo se han institucionalizado y no permiten la universalización del acceso a la educación; quiere decir que no podemos hablar de educación si no hablamos de 
acceso a la educación. Caso contrario convertimos al sistema educativo formal en una forma de exclusión para un porcentaje de la población. Eso demuestra la debilidad de la actoría del Estado en educación. Además, si lo básico no está cubierto, cómo podemos pasar a otro tipo de debate y otro tipo de educación.

Podemos llegar a satanizar este tipo de concepciones en el que nos proponemos cubrir lo mínimo necesario para la educación y podemos decir que eso no ingresa en el ámbito de las políticas, sino en el ámbito de la administración de los supuestos básicos para hacer educación. Mas el objetivo del planteamiento de este tipo de políticas se dirige a superar conflictos históricos de segregación a grupos y falta de acceso a la educación formal. Podemos lamentar que estemos en este punto de debate, pero toda política debe partir del contexto desde el cual trabajamos y el contexto ecuatoriano exige la universalización de la educación formal como una necesidad concreta que poseen los grupos sociales más desprotegidos y empobrecidos.

Por más elucubraciones que podamos hacer, nos queda apoyar los procesos de universalización de la educación, pues estamos en sociedades excluyentes, racistas y clasistas. El estar conscientes de que este tipo de políticas educativas que plantea el Estado están en los niveles de debate de la infraestructura mínima para tener educación, debe llevarnos a apoyarlas para que el país cubra esos mínimos y poder pasar al debate sobre la educación que queremos.

Por ahora el Estado nos plantea este reto en base a los mínimos, que son necesarios pero no suficientes. Además, no debemos olvidar que el Estado no es el único actor en las políticas públicas de la educación. 
Cada uno de los sujetos históricos colectivos e individuales generan procesos para inventar y reinventar la educación. Por ejemplo las familias, las comunidades y organizaciones negras, los municipios y las ciudades como tales, los grupos sociales organizados, los grupos culturales indígenas que poseen sus propias formas de transmitir y generar conocimiento. Esto solamente por colocar algunas posibilidades, pero existen miles de formas sociales que pueden constituirse en sujetos de la educación, en constructores de su propio proyecto educativo.

La sociedad, la comunidad, el país no terminan en el Estado, se encuentran mucho más "allá" del Estado. Y ese "más allá" del Estado debe también sacar sus propuestas y políticas educativas. Por ejemplo, pensemos en la ciudad como un actor que asume su reto de ser para la educación, la ciudad pensada como un mundo de creación humana para las presentes y futuras generaciones. Pensemos en la ciudad como "espacio" de aprendizaje.

Cada ciudad es historia, estética, política; su construcción física, sus imaginarios edificados están en cada muro, en cada avenida, en los edificios, en la forma de ser y de vivir de su gente. Los niños/as, jóvenes, adultos, migrantes y los ciudadanos mismos aprenden de aquello que es su ciudad. La ciudad es co-responsable del proceso educativo; ella ha de "hacerse" como espacio de aprendizaje y debe definir lugares específicos para el acto educativo: museos, zoológicos, bibliotecas, galerías, exposiciones, centros culturales, centros de investigación y eventos artísticos, sólo por nombrar algunos.

Por eso es importante afirmar que no basta con conocer que la ciudad es educativa, independientemente de nuestro querer o deseo. La ciudad se hace educativa por la necesidad de educar, de aprender, de enseñar, de conocer, 
de crear, de soñar, de imaginar con que todos nosotros, mujeres y hombres, impregnamos sus campos, sus montañas, sus valles, sus ríos, impregnamos sus calles, sus plazas, sus fuentes, sus casas, sus edificios, dejando en todo el sello de cierto tiempo, de estilo, de gusto de cierta época. La ciudad es cultura, creación, no solo por lo que hacemos en ella y con ella, sino también por la propia mirada estética o asombrada gratuita que le damos. La ciudad somos nosotros y nosotros somos la ciudad. Pero no podemos olvidar que lo que somos guarda algo que fue y que nos llega por la continuidad histórica -de la que no podemos escapar, pero sobre la cual podemos trabajar- y por las marcas culturales que heredamos.

En cuanto educadora, la ciudad es también educanda. Buena parte de su tarea educativa tiene relación directa con nuestra posición política y, obviamente, con la manera en que ejercemos el poder en la ciudad y el sueño o la utopía de que impregnamos la política, al servicio de qué y de quién la hacemos. La política de gasto público, la política cultural y educacional, la política de la salud la de los transportes, la del tiempo libre. La utopía política acerca de cómo subrayar tal o cual conjunto de memorias de la ciudad, a través de cuya sola existencia la ciudad ejerce su papel educativo. Hasta ahí, nuestra decisión política puede intervenir. ${ }^{3}$

Es triste ver que algunas ciudades en nuestros países latinoamericanos y sus gobiernos locales no asumen el reto de ser y tener espacios educativos. Ciudades que no contemplan ni trabajan su memoria histórica, sus instituciones y las expresiones de su propia gente.

Es necesario pensar la ciudad como "espacio" de y para la educación de las personas. Pero la sociedad como actor de las políticas educativas es tan diversa que puede partir de instancias menos institucionales, más complejas y desde actores sociales diferenciados. Veamos algunos ejemplos: 


\section{a. La comunidad educativa solidaria ${ }^{4}$}

Ésta es una experiencia que nace desde el Instituto Karl Popper de Quito, en trabajo conjunto con comunidades indígenas, gobiernos locales, escuelas unidocentes, universidades, entre otras instituciones. La figura de "Comunidad educativa solidaria" parte del mandato constitucional que considera que la educación es un "bien público" y tanto la Constitución de la República del Ecuador, como el espíritu ancestral de solidaridad comunitaria de nuestro pueblo, disponen que este preciado bien debe ser cuidado, protegido y acrecentado por todos y para todos los ecuatorianos.

Basa su organización en la voluntad de los miembros de cada comunidad y genera procesos diversos. Las comunidades establecen sus esquemas y compromisos en mutuos acuerdos y trabajan para mejorar la educación de su localidad. A la comunidad pueden pertenecer padres de familia, profesionales voluntarios, docentes de escuelas involucradas, familias, gobiernos locales, entre otros diversos actores. Cada comunidad educativa establece sus urgencias y políticas, pero mantienen interconexión para generar procesos de participación, capacitación, debate sobre la educación. No existe una organización jerárquica o diferenciada de poder, sino un esquema complejo y abierto para que los procesos se generen según las necesidades y capacidades.

Sus principios básicos están dados en la convicción de que la consecución de una sociedad más equitativa solamente se hará realidad si se logra formar, en todo lugar posible, un interés social superior por la educación. El interés superior sobre la educación debe manifestarse a través de la solidaridad, que implique el aporte generoso de conocimientos, competencias, experiencias, creatividad y capacidad de gestión, recursos e infraestructura de 
todos los miembros que conforman la comunidad educativa solidaria.

El interés superior de la comunidad es el de apoyar al mejoramiento de la calidad de la educación, especialmente en aquellos que tienen menos oportunidades, así como también el formar una innovadora cultura de protección a la educación local, a fin de que se mantenga vigente una noción de la calidad de vida y sus conexiones con la forma de hacer educación. Un interés primordial de la comunidad educativa solidaria es promover el desarrollo integral del maestro, su dignificación y respeto dentro de la comunidad.

Los miembros de una comunidad educativa solidaria aceptan su responsabilidad social y compromiso ético con el cuidado, protección y fortalecimiento de la educación. Asumen que la historia social integral de nuestro país se hace desde la educación, debe estar marcado por la afectividad y la solidaridad, busca la calidad de vida de las personas y los grupos, tiene por norte el "bien común".

Es una experiencia colectiva que retoma su responsabilidad con la educación y se auto-organiza desde la complejidad para abrir caminos a sus inquietudes, necesidades y conceptualizaciones.

\section{b. La escuela en casa}

Para poder sostener un enfoque de recrear las políticas y las dimensiones educativas se necesita continuamente inventar la educación, volverla a crear de acuerdo con las épocas y las construcciones teóricas que las sociedades construyen. Desde estas convicciones, algunos grupos populares se resisten a enviar a sus hijos e hijas a la educación formal y generan procesos diferenciados de educación desde la responsabilidad familiar o grupal. En algunas experiencias inicia una familia con la alternativa 
educativa y luego se van sumando otras familias con sus niños y niñas.

Las experiencias ya han recorrido años y se han convertido en verdaderas alternativas, a pesar de la falta de aprobación oficial del Estado y de los grupos sociales que rodean a las experiencias. Las iniciativas se han convertido en posibilidad de educar a los niños y niñas en la casa, y otras se han dado desde la circulación de los educandos en diferentes familias que se comprometen a formarlos en experticias que tienen los padres.

No podemos decir que esto es nuevo, ni que hay una renovación social en este sentido. Lo novedoso es que este tipo de propuesta anhela institucionalizarse como opción y validarse. No es sólo negar el sistema educativo, sino que presenta una posibilidad de educación para la sociedad. Las propuestas para "legalizarse" son varias y golpean las puertas oficiales. Cada vez las instancias del Estado se sienten más interrogadas por estas formas de presentarse de la educación alternativa.

Podemos trabajar infinidad de ejemplos, pero ésa no es la intención de este trabajo, sino mostrar a la comunidad que se apropia de la acción educativa, asume el reto de construir las políticas educativas desde sus propias dimensiones. Es importante ver el rol de la comunidad en el trabajo que realiza sobre sus propios sueños, anhelos e historia.

\subsection{Los llamados "medios de comunicación" 5 y las mediaciones comunicacionales}

En una sociedad marcada por las tecnologías de información y comunicación, en la que las herramientas para el uso de la palabra se han convertido en parte de la palabra misma, no podemos dejar fuera de la educación y de sus políticas el debate sobre el cómo pueden aportar los llamados "medios de comunicación" en la construc- 
ción de un proyecto de sociedad y por tanto en un proyecto de educación.

Partimos del supuesto de que los llamados "medios de comunicación", que en sentido comunicativo solamente son medios de información, son actores sociales, pues manejan la opinión como construcción de sentidos sociales que se elaboran desde ciertos intereses de grupo. Son actores sociales porque realizan actoría dentro de una comunidad local, nacional o internacional y, además, diseñan una sociedad en base a los sentidos que construyen en el manejo de la información.

Pero ¿qué tiene que ver esto con la educación? Pues nuestro debate solamente se concentrará en un punto: la necesidad de generar compromiso social educativo desde los medios de información y llegar a construir comunicación como mediación dialógica dentro de la sociedad.

Para la propuesta del párrafo anterior, recordemos primero que la comunicación es un bien público, por tanto debe ser considerado como tal y ponerse al servicio de los miembros de la comunidad, de la gente de una sociedad. Pero, a lo largo de toda Latinoamérica podemos ver que las frecuencias, propiedad del Estado, de radio y televisión y los permisos para la prensa escrita están manejadas, en su gran mayoría, por grupos de poder económico, político y social. Desde allí logran manejar discursos que favorecen a sus intereses. Así es imposible que asuman sus retos educativos.

Los medios de información están más comprometidos con el marketing y con hacer público el dolor y la angustia de la gente, que con un compromiso social de apoyo a procesos educativos. Podemos ver diarios que trabajan con el morbo sobre el sufrimiento de la gente, junto a la sexualidad grotesca dejar desde imágenes de cuerpos de mujeres o de niños; canales de televisión que exponen la sobreexplotación machista y burlona de lo femenino; la 
llamada prensa roja y/o amarilla entre otras realidades que se edifican como sensibilidades.

Además, los llamados medios de comunicación se han apoderado de la palabra y han sublimado la palabra individual. La superación de lo individual desde las propuestas comunitarias y colectivas -que son herencia ancestral de nuestros pueblos- exige que en toda posesión de la palabra esté presente la responsabilidad social. Por tanto no se trata de que cada individuo piense y exprese desde su individualidad, sino que se construya la posesión de la palabra como acto democrático y participativo.

En cuanto a la educación, es necesaria una legislación que exija la participación de los medios de manera comprometida en programas anuales educativos y que sea una política permanente. No sólo se trata de pasar algunos documentales, sino toda una producción desde Latinoamérica y Ecuador. Además, los mismos medios deben autorregularse y lograr mejores niveles de autocontrol. La posibilidad de una defensoría interna de los medios puede ayudar a depurar su acción social.

Junto a la acción en los medios, es necesaria la acción de la educación: es hora de enseñar a nuestros niños y niñas a leer la imagen; a poder interpretar mensajes visuales. Junto a esto la filosofía tiene una larga tarea de conceptualizar las nuevas tecnologías de información y comunicación como parte de la existencia integral y cultural de la humanidad.

Si queremos avanzar es necesario pensar la educación como un acto comunicativo y eso supera el simple trabajo con los medios de la información, puesto que requiere entender la comunicación como ontología de lo humano, como construcción necesaria para ser y hacerse humano en relación a la palabra mía y del otro. La comunicación en definitiva es mediación inter-personal. Y la mediación no es posible fuera del diálogo. 
Ahora bien, dentro de diversos sectores sociales existe una gran preocupación por la propuesta de diálogo para lo educativo y para el proceso educativo. El diálogo es uno de los mayores acuerdos enunciados en las políticas educativas, por parte de los actores.

Pero para construir ese diálogo se necesita realizar lecturas de la realidad para entenderla y recrearla. Lecturas que reconstruyan los sentidos planteados por los sectores dominantes y neocoloniales. Lecturas que puedan ir más allá de lo observable, que ingresen en lo profundo de los sentidos construidos sobre lo humano.

El diálogo exige la participación de un "yo" y unos "otros" que se comunican en igualdad de condiciones, en respeto mutuo, en reconocimiento. De ahí podemos sostener que las concepciones de una sociedad sobre los otros, la alteridad es importante para construir diálogos. La dialéctica de la negación del otro de entrada no permite crear espacios de diálogo.

Si la sociedad del conocimiento desafía a ampliar nuestra cosmovisión y a abrir nuestra sensibilidad, esta presencia del otro debería constituir un activo potenciable. $\mathrm{Si}$ en lugar de negar la identidad del otro, la reconocemos incluso presente dentro de nosotros, nuestra cosmovisión se expande. El mundo no se nos derrumba si nos abrimos a la identidad en la diferencia, sino que se enriquece con nuevos contenidos. Esto significa no sólo ampliar nuestra percepción del mundo, sino que también se traduce en efectos prácticos tales como: adecuaciones tecnológicas, conocimiento e información sobre tecnologías disponibles y asimilación creciente y adecuada de las mismas; o desarrollar vínculos comunitarios que pueden fortalecer la democracia social y enriquecer los lazos de pertenencia y comunicación. ${ }^{6}$

El diálogo ${ }^{7}$ se construye desde los procesos, no desde los ideales de perfección. No podemos pensar en un 
ideal, en el "deber ser" de la educación y desde allí entrar a dialogar, porque eso nos lleva al discurso vacío, a la inexistencia de posibilidades reales de construir un estado dialogal. Si el ideal es primero y el deber ser se impone, entonces ya no hay nada que decir, sólo queda obedecer y caminar hacia el ideal. El "deber ser" de la educación es un sistema de dominación. Mucho más si ese "deber ser" viene dado por un pequeño grupo que establece lo que hay que hacer.

El diálogo debe remontarse al reconocimiento de la diferencia. Es fundamental para posibilitar el diálogo como eje de la educación sacar de la mente y de los cuerpos de los sujetos históricos las ideas homogeneizantes y las verdades puras, para permitir diversidad en un manantial de posibilidades que la misma vida nos ofrece.

\section{La necesidad de pensar políticas de educación desde la diversidad}

La educación ha aportado al fatal destino homogenizador, cuando ha construido mentalidades que sólo pueden concebir conocimientos homogéneos y mecánicos. Mentalidades que no pueden soportar la diferencia, ni siquiera de ideas, mucho menos de hechos que se consideran vitales. La escuela ha trabajado dentro de la igualdad: niños iguales, niñas iguales, trato igual para todos, iguales procesos, iguales cobros, iguales uniformes, iguales zapatos, iguales ejercicios matemáticos, iguales notas para iguales resultados. Es un orgullo institucional tratar a todos por igual. En una sociedad de diferencias, la igualdad es un valor: fatal contradicción que genera exclusiones. Porque si tenemos iguales cobros para una escuela cara, ya sabemos quienes van a ser iguales y quienes quedan excluidos. Porque si tenemos igualdad en la escuela de varones, ya sabemos que las mujeres quedan excluidas de esa igualdad. 
Algunas metodologías y didácticas educativas están construidas desde la igualdad de procesos y contextos. Olvidan las diferencias en un mar de imposibilidades. Es imposible tratar a niños y niñas desde sus diferencias, eso sería "injusto". La "justicia" establece que se debe tratar igual a todos. Ese tipo de trabajo educativo construye mentalidades que siempre manejan procesos homogenizadores. Y consideran que es un avance homogenizar las particularidades. Una particularidad que se construye como "buena" pasa a ser un universal obligado. Y ese siempre es el proceso de construcción social.

En sociedades como las nuestras se vuelve una exigencia construir políticas desde la diferencia, pues somos pueblos marcados históricamente y ontológicamente por las diferencias: de género, de cultura o etnia, de clase, de edad, de ideología, entre muchas otras.

\section{A manera de conclusión: la educación como proyecto}

El ser humano y su capacidad de educarse para construir su humanidad es temporal, es un proyecto inacabado. Por ello la educación sólo es en cuanto se hace. No hay posibilidad de tener entendido y planificado todo para de ahí darlo o ejecutarlo. No se puede tener algo completamente listo, algo acabado en sentido estricto para transmitirlo a los demás, porque la educción es un proceso. La planificación es posible en la medida que cuente con la fatalidad de la improvisación y de la necesidad de la revisión permanente. Además, es necesario colocar los sueños de una colectividad, el deseo de otro mundo posible dentro de la planificación. Por ello siempre será necesario planificar bien, para improvisar mejor.

El manejo de las viejas certezas, las categorías de siempre ya no ayudan a recrear la educación para nosotros 
y para las futuras generaciones, porque los contextos han cambiado y porque los sentidos que construimos sobre el mundo y sobre la vida son diferentes existencialmente.

Es hora de abandonar los miedos para avanzar desde la esperanza. Las grandes verdades absolutas y objetivas que teníamos para estar seguros, ahora se han convertido en nuestra mayor inseguridad. La posibilidad de inventar y reinventar las categorías en la educación parecen lejanas, pero son necesarias.

\section{Notas}

1 Cfr., Fundación Mirarte, Sistematización proyecto "Jugando con el abuelo", Otavalo, 2007.

2 Misterio e Educación y Cultura, Plan decenal de Educación del Ecucación del Ecuador, 2006 - 2015.

3 Freire Paulo, Política y Educación, México D.F., Siglo veintiuno editores, 1999, p. 26-27.

4 Cfr., Moreno Beatriz, Base conceptual del estatuto de formación de "Comunidad educativa solidaria", Quito, Instituto Karl Popper, 2006. Documento versión digital.

5 Partimos de las diferencias que se realizan en diversas teorías de la comunicación, entre medios de información y mediaciones de la comunicación. Utilizamos sin embargo los términos "medios de comunicación” por ser el más enunciado fuera de los ámbitos académicos.

6 Calderón, Hopenhayn, Ottone, Esa esquiva modernidad. Desarrollo, ciudadanía y cultura en América Latina y el Caribe, Caracas, Nueva Sociedad, , 1996, p. 80.

7 Cfr., Reyes Darwin, La construcción de la interculturalidad desde las políticas educativas en la experiencia del sistema de formación docente en el Programa Académico Cotopaxi, de la UPS (Tesis), Universidad Salesiana, Quito, 2006.

\section{Bibliografía}

Álvarez Freddy

1999 Las derivas de la alteridad, Quito, Efímera. 
Álvarez Freddy y otros

2005 El arte de cambiar las personas que cambian las cosas, Quito, Red Nuevo Paradigma.

Auge Marc

2000 La guerra de los sueños, Madrid, Gedisa.

Moreno Beatriz

2006 Base conceptual del estatuto de formación de "Comunidad educativa solidaria”, Quito, Instituto Karl Popper.

Calderón, Hopenhayn, Ottone

1996 Esa esquiva modernidad. Desarrollo, ciudadanía y cultura en América Latina y el Caribe, Caracas, Nueva Sociedad.

De Souza Silva y otros

2005 La innovación de la innovación institucional, Quito, Red Nuevo Paradigma.

Esposito Roberto

2006 Comunitas. Origen y destino de la comunidad, Buenos Aires, Amorrortu.

Freire Paulo

1999 Política y Educación; México D.F., Siglo veintiuno editores.

Fundación Mirarte

2007 Sistematización proyecto "Jugando con el abuelo". Otavalo.

Homi Bhabha

2002 El lugar de la cultura, Buenos Aires, Manantial.

Morín, Edgar

2002 La cabeza bien puesta: Repensar la reforma, reformar el pensamiento: bases para una reforma educativa, Buenos Aires, Nueva Visión.

Morin Edgar, Roger Emilio y Motta Raúl

2003 Educar en la era planetaria, Barcelona, Gedisa.

Ministerio de Educación y Cultura

2006 Plan decenal de Educación del Ecuador 2006 - 2007.

Quijano Aníbal

2001 Colonialidad del poder. Cultura y conocimiento en América Latina, en: Capitalismo y geopolítica del conocimiento: El euro centrismo y la filosofía de la liberación en el debate intelectual contemporáneo. Mignolo Walter (compilador), Buenos Aires, Ediciones del signo. 